\title{
Distinct microbes, metabolites, and ecologies define the microbiome in deficient and proficient mismatch repair colorectal cancers
}

Vanessa L. Hale ${ }^{1,2,3 \dagger}$, Patricio Jeraldo ${ }^{2,3 \dagger}$, Jun Chen ${ }^{2,4}$, Michael Mundy ${ }^{5}$, Janet Yao ${ }^{2}$, Sambhawa Priya ${ }^{6}$, Gary Keeney ${ }^{7}$, Kelly Lyke ${ }^{3}$, Jason Ridlon ${ }^{8}$, Bryan A. White ${ }^{8}$, Amy J. French? ${ }^{7}$ Stephen N. Thibodeau', Christian Diener ${ }^{9}$, Osbaldo Resendis-Antonio ${ }^{9,10}$, Jaime Gransee ${ }^{11}$, Tumpa Dutta ${ }^{11}$, Xuan-Mai Petterson ${ }^{11}$, Jaeyun Sung ${ }^{2,3}$, Ran Blekhman ${ }^{6}$, Lisa Boardman ${ }^{12}$, David Larson ${ }^{13}$, Heidi Nelson ${ }^{2,13}$ and Nicholas Chia ${ }^{2,3^{*}}$ (D)

\begin{abstract}
Background: Links between colorectal cancer (CRC) and the gut microbiome have been established, but the specific microbial species and their role in carcinogenesis remain an active area of inquiry. Our understanding would be enhanced by better accounting for tumor subtype, microbial community interactions, metabolism, and ecology.

Methods: We collected paired colon tumor and normal-adjacent tissue and mucosa samples from 83 individuals who underwent partial or total colectomies for CRC. Mismatch repair (MMR) status was determined in each tumor sample and classified as either deficient MMR (dMMR) or proficient MMR (pMMR) tumor subtypes. Samples underwent 165 rRNA gene sequencing and a subset of samples from 50 individuals were submitted for targeted metabolomic analysis to quantify amino acids and short-chain fatty acids. A PERMANOVA was used to identify the biological variables that explained variance within the microbial communities. $\mathrm{dMMR}$ and PMMR microbial communities were then analyzed separately using a generalized linear mixed effects model that accounted for MMR status, sample location, intra-subject variability, and read depth. Genome-scale metabolic models were then used to generate microbial interaction networks for dMMR and pMMR microbial communities. We assessed global network properties as well as the metabolic influence of each microbe within the $\mathrm{dMMR}$ and pMMR networks.
\end{abstract}

Results: We demonstrate distinct roles for microbes in dMMR and PMMR CRC. Bacteroides fragilis and sulfidogenic Fusobacterium nucleatum were significantly enriched in $\mathrm{AMMR} C R C$, but not PMMR CRC. These findings were further supported by metabolic modeling and metabolomics indicating suppression of $B$. fragilis in PMMR CRC and increased production of amino acid proxies for hydrogen sulfide in dMMR CRC.

Conclusions: Integrating tumor biology and microbial ecology highlighted distinct microbial, metabolic, and ecological properties unique to dMMR and PMMR CRC. This approach could critically improve our ability to define, predict, prevent, and treat colorectal cancers.

\footnotetext{
* Correspondence: chia.nicholas@mayo.edu

${ }^{\dagger}$ Vanessa L. Hale and Patricio Jeraldo contributed equally to this work.

${ }^{2}$ Microbiome Program, Center for Individualized Medicine, Mayo Clinic,

Rochester, MN, USA

${ }^{3}$ Division of Surgical Research, Department of Surgery, Mayo Clinic,

Rochester, MN, USA

Full list of author information is available at the end of the article
}

(c) The Author(s). 2018 Open Access This article is distributed under the terms of the Creative Commons Attribution 4.0 International License (http://creativecommons.org/licenses/by/4.0/), which permits unrestricted use, distribution, and reproduction in any medium, provided you give appropriate credit to the original author(s) and the source, provide a link to the Creative Commons license, and indicate if changes were made. The Creative Commons Public Domain Dedication waiver (http://creativecommons.org/publicdomain/zero/1.0/) applies to the data made available in this article, unless otherwise stated. 


\section{Background}

The gut microbiota has been linked to colorectal cancer (CRC) in many studies [1-9] and serves as a very promising target for diagnostic, prophylactic, and therapeutic applications. Yet, despite intense study, only a few microbial species-like Fusobacterium species-are consistently observed across studies [10-14], while many microbial associations appear to be cohort-specific. Meta-analyses have attempted to overcome the limited statistical power of smaller studies [15] but are limited by the strong biases introduced through varying collection, sequencing, and data processing methodologies [16]. Mechanistic studies in mouse models have identified strong causative links between specific microbes (e.g., Fusobacterium nucleatum, Bacteroides fragilis) and CRC development and progression $[11,17-24]$, but these models have limited applicability in genetically diverse human populations. Capturing some of this genetic diversity, on the other hand, may improve our ability to discriminate tumor and normal microbial communities and more clearly define pathways to CRC.

One genetic subtype of CRC is based on the presence or absence of mutations in the DNA mismatch repair system. This system involves multiple protein complexes that recognize, remove, and correct mismatched DNA base pairs. Mutations in these protein complexes can render the mismatch repair system defunct-allowing mutations to accumulate. This hypermutable subtype is known as deficient mismatch repair (dMMR) and occurs in approximately $15 \%$ of sporadic CRCs [25]. CRCs that do not exhibit mutations in the mismatch repair system are known as proficient mismatch repair (pMMR) CRCs [26]. In general, dMMR CRCs are microsatellite instable (MSI-H), hypermethylated, and associated with BRAF V600E mutations and low nuclear beta-catenin expression, whereas pMMR CRCs are more commonly microsatellite stable (MSS) and associated with KRAS mutations [27, 28]. Clinically, MMR status is associated with patient prognosis and age, as well as tumor location and stage: Specifically, $\mathrm{dMMR}$ CRCs have a better prognosis and occur more often on the right side of the colon in older patients with early-stage CRC [26]. Finally, dMMR and pMMR CRC not only have different endpoints, but may also have different paths to tumorigenesis [29] as supported by emerging evidence that dMMR CRC arises from sessile serrated adenomas [30] as opposed to the more classic tubular adenoma associated with pMMR CRC [30].

The distinct phenotype of dMMR CRC suggests that host-and possibly also microbial-dynamics are greatly altered in association with deficient mismatch repair. Previous work has examined the role of other differentiating factors in the CRC microbiome including location [31], MSS/MSI status [12], and consensus molecular subtypes [32]. However few CRC microbiome studies account for MMR status [32-34] or microbial dynamics
[35], and no studies, to our knowledge, have assessed both MMR status and microbial community dynamics. In addition, our study examines demographic, genetic, and tumor features together in a relatively large prospectively collected cohort.

Here, we undertook a new approach in a study involving 83 patients who underwent partial or total colectomy for CRC. From each patient, we collected colon tissue and mucosal samples at tumor and normal-adjacent sites. MMR status was extracted from patient records or determined by testing formalin-fixed paraffin-embedded tumor tissue for the expression of four MMR proteins (MLH1, MSH2, MSH6, PMS2). From this, patient tumors were characterized as either deficient (dMMR) or proficient (pMMR) mismatch repair. Microbial composition was assessed via $16 \mathrm{~S}$ rRNA gene sequencing. A subset of colon tissue samples additionally underwent targeted metabolomic analysis to quantify amino acids. A portion of these data was published previously [35] in a study that highlighted the value of integrating in silico genome-scale metabolic model predictions and in vivo experimental metabolomic data.

From these data, we assessed the relative importance of MMR status compared to other biological factors reported to alter the microbiome [36]. MMR status was the strongest predictor of microbial community variance in comparison to sample location (proximal/distal and on/off tumor), body mass index (BMI), age, and sex. Separate analyses of the $\mathrm{AMMR}$ and $\mathrm{PMMR}$ microbial communities revealed that many common CRC-associated microbial signatures [15]-including Fusobacterium nucleatum, Fusobacterium periodonticum, and Bacteroides fragiliswere all enriched in dMMR but not pMMR tumors. Functional differences were examined using a combination of metabolomics and community metabolic modeling. Our results indicate greater hydrogen sulfide production (inferred through amino acid proxies) in dMMR CRC and greater metabolic suppression of $B$. fragilis in pMMR CRC. Our work demonstrates distinct microbial, metabolic, and ecological attributes of dMMR and pMMR microbial communities, serving to further emphasize the importance of considering tumor biology and microbial interactions in studies of the CRC microbiome.

\section{Methods}

\section{Human subject enrollment}

Adults (older than 18 years old) who were determined to be candidates for colorectal cancer surgery were voluntarily enrolled at Mayo Clinic in Rochester, Minnesota. Exclusion criteria included chemotherapy or radiation in the 2 weeks leading up to enrollment. Total or partial colectomies were performed on every patient, and colon tissue and mucosal samples were collected from tumor and normal-adjacent sites. Sample location was defined as follows: "proximal" samples were derived from the cecum and ascending colon. 
"Distal" samples were derived from the transverse, descending, or sigmoid colon, or rectum. MMR status was determined in 83 patients: 25 had dMMR CRC and 58 had pMMR CRC (Table 1). We used univariable logistic regression ( $\mathrm{R}$ v3.1.2) to compare demographic (age, sex, BMI, smoking history) and tumor features (location and stage) between $\mathrm{dMMR}$ and $\mathrm{pMMR}$ groups.

\section{MMR status determination}

Mismatch repair (MMR) pathway and microsatellite instability (MSI) test results were extracted from patient records if available. For patients without MMR test results, banked formalin-fixed paraffin-embedded colon tumor tissue blocks were submitted to the Mayo Clinic Pathology Resource Core for sectioning into $10-\mu \mathrm{m}$-thick slices. Slices were then submitted to the Mayo Clinic Molecular Genetics Laboratory for immunohistochemistry staining of MMR proteins (MLH1, PMS2, MSH2, MSH6).

\section{S DNA extraction, sequencing, and sequence processing}

DNA extraction [37] and library preparation on colon tissue (tumor and normal-adjacent) and mucosa were performed as described previously in the Mayo Clinic Microbiome Laboratory [35]. Samples were submitted for $16 \mathrm{~S}$ rRNA gene sequencing (V3-V5 region) at the Mayo Clinic Medical Genomics Facility (Illumina MiSeq, $2 \times 300$, 600 cycles, Illumina Inc.). Sequencing yielded a total of 41,400,384 reads with a median of 70,208 reads per sample. Reads

Table 1 Demographic and tumor features of individuals identified as having $\mathrm{dMMR}$ or PMMR CRC

\begin{tabular}{|c|c|c|c|}
\hline & $\mathrm{dMMR}$ & pMMR & $p$ value \\
\hline \multicolumn{4}{|l|}{ Sex, $n(\%)$} \\
\hline Male & $10(40)$ & $34(59)$ & \multirow[t]{2}{*}{0.122} \\
\hline Female & $15(60)$ & $24(41)$ & \\
\hline \multicolumn{4}{|l|}{ Age, years } \\
\hline Mean (SD) & $74(18)$ & $63(13)$ & \multirow[t]{2}{*}{0.002} \\
\hline Range & $23-95$ & 33-90 & \\
\hline BMI (SD) & $27(5)$ & $29(8)$ & 0.273 \\
\hline \multicolumn{4}{|l|}{ Smoke ever? n (\%) } \\
\hline Yes & $13(52)$ & $28(48)$ & \multirow[t]{2}{*}{0.982} \\
\hline No & $12(48)$ & $30(52)$ & \\
\hline \multicolumn{4}{|c|}{ Tumor location, $n$ (\%) } \\
\hline Proximal colon & $18(72)$ & $14(24)$ & \multirow{3}{*}{$\begin{array}{l}p<0.0001 \text { between } \\
\text { proximal and distal }\end{array}$} \\
\hline Distal colon & $7(28)$ & $43(74)$ & \\
\hline Both & 0 & $1(3)$ & \\
\hline \multicolumn{4}{|l|}{ Stage, $n(\%)$} \\
\hline Early (1-2) & $18(72)$ & $22(38)$ & \multirow{3}{*}{$\begin{array}{l}0.0007 \text { between } \\
\text { early and late }\end{array}$} \\
\hline Late (3-4) & $4(16)$ & $33(57)$ & \\
\hline Stage unknown & $3(12)$ & $3(5)$ & \\
\hline
\end{tabular}

were processed using DADA2 v1.6 to obtain error-corrected amplicon sequence variant representatives-analogous to operational taxonomic units with single-nucleotide resolution (sOTUs) [38]. sOTUs were annotated with genus-level taxonomy using the RDP Naïve Bayesian Classifier [39] as implemented in DADA2 and, if possible, to species level using DADA2, both against the SILVA 16S database, v132 [40]. sOTUs annotated as chloroplast and mitochondria were removed. Resulting sOTUs were filtered for possible non-specific amplification using SortMeRNA v2.0 [41] and Infernal v1.1.2 [42]. sOTUs with fewer than 10 reads across all samples were excluded. Multiple sequence alignment of the sOTUs was performed using Infernal v1.1.2 [42], and an approximate Maximum Likelihood phylogeny was calculated using FastTree v2.1.9 [43].

Statistical analyses of $16 \mathrm{~S}$ rRNA microbial community data UniFrac distance matrices [44] based on the microbial communities in all samples were generated using the phyloseq [45] package v1.22.3. A permutational multivariate analysis of variance (PERMANOVA) was then performed on the distance matrix to assess the effects of MMR status and sample location (proximal/distal and on/off tumor) on variance between microbial communities. The PERMANOVA additionally accounted for subject age, sex, BMI, and sample type (mucosa versus colon tissue) and was performed based on the adonis function in the vegan [46] package v2.5-1, with 999 permutations. Different permutation schemes were used to maintain the original correlation structure when testing the significance of relevant variables.

A generalized linear mixed model (GLMM) [47] was calculated for each sOTU to estimate its abundance (read counts) in relation to predictors that included MMR status and sample location (proximal/distal and on/off tumor). Models were corrected for subject intervariability, specimen type (mucosal vs tissue biopsy), and sequencing read depth, allowing for interactions. We used the package glmmTMB [48] v0.1.4 to estimate the abundance of each microbe under a zero-inflated Poisson distribution. For each predictor, sOTUs were excluded where the method did not converge or the Akaike Information Criterion (AIC) for model quality was not defined. Multiple hypothesis correction was calculated using the Benjamini-Hochberg procedure.

\section{Validation of differentially abundant microbes using an independent cohort}

To validate the differentially abundant microbes associated with $\mathrm{dMMR}$ status, we investigated data from a recent study that included microbiome profiling in tumor and matched normal tissue samples in 44 CRC patients [49]. Individuals with microsatellite instable (MSI-H) tumors or downregulation of any of the 4 MMR genes 
(MLH1, MSH2, MSH6 and PMS2)-as assessed using RNA-Seq-were categorized as dMMR. A cutoff of $\log 2(-$ normal/tumor) $\geq 1$ was used to call a gene as downregulated in tumor. Individuals with microsatellite stable (MSS) tumors were categorized as pMMR. Altogether, we identified 9/44 patients as dMMR and the remaining 35/ 44 as pMMR. Using the $16 \mathrm{~S}$ rRNA gene to characterize these samples (as described in detail in [49]), we identified sOTUs associated with dMMR tumor/normal and pMMR tumor/normal conditions. We first filtered rare sOTUs, only preserving sOTUs found in at least $50 \%$ of our samples, and then performed differential abundance analysis using phyloseq [45] (which uses DESeq2 to build negative binomial generalized linear models). We used the Benjamini-Hochberg method to control for the false discovery rate (FDR).

\section{Real-time PCR for the Bacteroides fragilis toxin gene}

Real-time PCR was performed as described previously [35] to test colon tissue and mucosal samples for the presence of the Bacteroides fragilis toxin (BFT) genes in the 22 $\mathrm{dMMR}$ individuals and 53 pMMR individuals. Primers included: BFT-F (5'-GGATAAGCGTACTAAAATACAGCT GGAT-3'), BFT-R (5' -CTGCGAACTCATCTCCCAGTAT AAA- $\left.3^{\prime}\right)$, and the probe (5'-FAM-CAGACGGACATTCT C-NFQ-MGB-3') [19].

\section{Modeling microbial hydrogen sulfide production}

We predicted hydrogen sulfide production within $\mathrm{dMMR}$ and pMMR tumor and normal-associated microbial communities as described previously [35]. Briefly, we aligned 16S rRNA gene sequences for dMMR tumor and normal samples (colon tissue and mucosa) and pMMR tumor and normal samples against complete genomes in PATRIC and then generated genome-scale metabolic models of each microbe (Additional file 1: Table S1). Genome-scale metabolic models use gene annotations from a microbial genome to predict the metabolic inputs and outputs of that microbe. To predict how a microbe might interact within a community, we used MICOM, an open-source platform to assess microbial community metabolism (https://github.com/resendislab/micom). Specifically, we used flux balance analysis with MICOM's community growth objective and constraint formulation in order to evaluate hydrogen sulfide flux as a measure of hydrogen sulfide production within each microbial community.

\section{Microbial influence network}

To select sOTUs for the Microbial Influence Networks (MINs), we used GLMM results to choose tumor and normal-associated microbes in dMMR and pMMR samples with a linear effect size greater than 0.25 , regardless of statistical significance. Effect size captures biological impact potential while significance measures certainty.
In this case, we wanted to assess the metabolic influence (i.e., biological impact) of microbes in relation to their respective microbial communities; as such, it was more appropriate to filter by effect size. For each sOTU, the $16 \mathrm{~S}$ rRNA gene consensus sequence was aligned against complete genome in the PATRIC system using VSEARCH v2.7.1, with a minimum nucleotide identity of $90 \%$. When this procedure generated multiple top hits, we selected a genome, in order, to the most complete genome (fewer contigs), a type strain, a strain with a binomial name, and the closest match to the $16 \mathrm{~S}$ taxonomy (when possible). For each genome, we then reconstructed and downloaded its corresponding genome-scale metabolic model using the PATRIC service. When sOTUs mapped to the same model, we used that model only once, effectively merging those sOTUs in further analysis, with an exception for when two sOTUs were associated with opposite conditions (i.e., tumor and normal-adjacent samples), in which case, we discarded that model from further consideration. The decision to discard was also based on the observation that low identity hits or sOTUs with taxonomy not sufficiently resolved were typically involved in these few cases.

After obtaining the genome-scale metabolic models (GEMs), we calculated "growth" on complete media with no oxygen. This was done by calculating optimal metabolic reaction fluxes using a Flux Balance Analysis [50], in which "growth" is the calculated flux of the reaction defining biomass for a microbe. We did this using a tool for assessing microbial metabolic interactions (MMinte) which evaluates the growth of microbes alone and when paired with another microbe [51]. Once single and paired growth values were calculated using the objective function given by MMinte [51], these values were then used to calculate the influence score. The influence score, $\alpha_{\mathrm{xm}}$, for a species, $\mathrm{m}$, with a different species $\mathrm{x}$ was calculated as

$$
\alpha_{x m}=g(x \mid m)-g(x)
$$

where $g(\mathrm{x})$ was the growth rate of $x$ alone and $g(\mathrm{x} \mid m)$ was the growth rate of $x$ in a community composed of both $\mathrm{x}$ and $\mathrm{m}$. Based on these scores, we then calculated the influence of each individual microbial model on the other microbes in the community as the sum of the absolute values of the differences in growth rates when paired with species $m$,

$$
G_{m}=\sum_{j}\left|\alpha_{j m}\right|=\sum_{j}|g(j \mid m)-g(j)|
$$

This scoring closely follows the spirit of the scoring from the global metabolic interaction modeling in Sung et al. [52]; to derive interactions, we used growth rates that were computationally inferred from comprehensive metabolic models in contrast to using experimentally verified transport reactions from a limited number of 
microbes and metabolites. Metabolic modeling based on flux balance analysis, as described here, provides a means to calculate a rate of steady-state growth, as normalized per unit mass, allowing us to take a simple sum in order to calculate influence under anaerobic conditions.

The percentage of negative interactions was calculated by counting the number of negative interactions over the number of total interactions in each microbial influence network (MIN). Statistical significance was based on the probability of getting equivalent results in dMMR and pMMR networks using the measured distributions of negative and positive interactions in each network and a scheme of random selection with replacement.

Finally, the resulting MIN [52] was visualized using Cytoscape v3.6.1 [53] with node size and edge weights set according to influence score and influence, respectively. The entire list of microbial influences in dMMR and pMMR subjects (Additional file 1: Tables S8 and S9) are too dense for direct visualization, and therefore, only a part of them are presented. More specifically, interactions below an influence of 10 in the case of both dMMR and pMMR were excluded. Unconnected nodes that had no influence were not included in the visualization.

\section{Estimating the effect of whole-community metabolic interactions on growth suppression}

In order to assess the degree to which a microbial species is suppressed by other members of the microbial community, we evaluate the interactions of the rest of the microbial community on a target member. It is worth emphasizing that this infers the effect of all members of a microbial community, in contrast to the MIN, which focuses on the tumor and normal-adjacent enriched microbes in dMMR and PMMR CRC. Briefly, the target organism's net interactions $S_{m}$ with each microbe in a given community will be calculated according to:

$$
S_{m}=\sum_{j} A_{j} \alpha_{m j}=\sum_{j} A_{j}(g(m \mid j)-g(m))
$$

i.e., the abundance-weighted sum of the metabolic influences on microbe $m$. When this sum is negative (as would be generally true in eubiosis), this yields a suppression score that reflects the magnitude of the negative interactions affecting microbe $\mathrm{m}$. For the purpose of this calculation, we calculate this in every sample, we use anaerobic conditions and only consider microbial species that make up greater than $5 \%$ of the relative abundance of the community in at least one sample, ensuring we do not miss any microbes that may have a significant effect on the suppression score.

\section{Results}

dMMR tumors associated with older-age and early-stage, proximal tumors

A total of 25 individuals with dMMR CRC and 58 individuals with pMMR CRC were involved in this study. Individuals with $\mathrm{dMMR}$ CRC were significantly older than individuals with pMMR CRC and significantly more likely to have an early-stage, proximal tumor (Table 1) in alignment with other studies on dMMR CRC [25]. Thus, to address potential confounding effects due to age and sample location (proximal/distal), we adjusted these variables in subsequent analyses.

\section{Tumor MMR status explains the largest variance between} microbial communities

To assess factors that contributed to variance in the microbial community data, we performed a PERMANOVA analysis on unweighted UniFrac distances between microbial communities in each sample. We included MMR status, sample location (proximal/distal, on/off tumor), age, sex, BMI, and sample type (colon tissue vs. mucosa) as potential predictors of the variance. We used both marginal and adjusted analyses where we included only a single factor in our assessment of percent variance explained or after correcting for all other factors, respectively. The adjusted analysis controlled the effects of other variables, and the resulting percent variance explained was independent of other variables and thus not subject to the confounding by the correlated variables. Remarkably, we found that MMR status explained more of the variance than any of the other 6 variables in both cases (Table 2), and it remained significant after adjusting for other variables $(p=0.004)$, indicating MMR status was independently associated with the microbiome composition. The difference between tumor and normal-adjacent samples was also highly significant $(p$ $<0.001$ from adjusted analysis; Table 2), indicating that the tumor samples harbor a unique microbiome. Moreover, when comparing the tumor-to-normal UniFrac distance between MMR subtypes (Additional file 1: Figure S1), the distance in the dMMR subtype was significantly larger than that in the pMMR subtype $(p=0.004)$, which suggests a potential stronger perturbation of the normal microbiome in the dMMR subtype.

\section{Distinct microbial communities associated with pMMR and $\mathrm{dMMR}$ tumors}

Given both the importance of MMR status to microbial community variance (Table 2) and the difference in tumor to normal UniFrac distances by MMR subtype (Additional file 1: Figure S1), we opted to assess microbial abundances in tumor and normal samples for each MMR subtype independently. We identified multiple differentially abundant sOTUs in dMMR and pMMR tumor samples as compared to normal-adjacent samples 
Table 2 Factors contributing to variance between microbial communities

\begin{tabular}{|c|c|c|c|c|}
\hline & Marginal & & Adjusted & \\
\hline Factors & $\%$ Variation & $p$ value & $\%$ Variation & $\overline{p \text { value }}$ \\
\hline MMR status & 2.58 & 0.001 & 1.85 & 0.004 \\
\hline Sample location_proximal/distal & 1.87 & 0.011 & 1.44 & 0.019 \\
\hline Sample type & 1.70 & 0.001 & 1.36 & 0.001 \\
\hline Sample location—on/off tumor & 1.50 & 0.001 & 1.01 & 0.001 \\
\hline Sex & 1.48 & 0.051 & 1.14 & 0.184 \\
\hline BMI & 1.34 & 0.108 & 1.60 & 0.025 \\
\hline Age & 0.96 & 0.542 & 1.02 & 0.319 \\
\hline
\end{tabular}

Percent variation and $p$ values in the first two columns were from marginal analyses (i.e., not adjusted for other factors). Percent variation and $p$ values in the last two columns were from analyses adjusting for all factors. Permutation tests (999 permutations) were used to calculate the $p$ values. For "Sample type" and "on/off tumor" factors, the permutation was confined within the subject. For the rest of the factors, the subjects were the permutation units (i.e., randomly assign a value to each subject) to account for the within-subject correlations

using a generalized linear mixed model (GLMM) that accounted for sample location (proximal/distal), sample type, and intrasubject sample correlation (Fig. 1; Additional file 1: Figure S2 (Venn diagram showing counts of microbes in each group); Additional file 1: Table S2 (list of microbes enriched in $\mathrm{dMMR}$ and $\mathrm{pMMR}$ tumor and normal-adjacent samples); Additional file 1: Table S3 (microbes enriched in dMMR tumors); Additional file 1: Table S4 (microbes enriched in pMMR tumors); Additional file 1: Table S5 (microbes enriched in the proximal or distal colon of individuals with dMMR CRC); Additional file 1: Table S6 (microbes enriched in the proximal or distal colon of individuals with pMMR $\mathrm{CRC})$ ). Only one microbe-Dorea longicatena-was significantly enriched in both $\mathrm{dMMR}$ and pMMR tumor samples. Four microbes had opposite associations with tumor or normal samples depending on MMR status: Faecalibacterium prausnitzii A2-165 and Blautia sp. Marseille-P2398 were significantly enriched in pMMR tumor and dMMR normal samples; Coprococcus comes ATCC 27758 and Bacteroides massiliensis B84634 were significantly enriched in dMMR tumor and pMMR normal samples. Notably, Fusobacterium and Bacteroides fragilis-microbes commonly associated with CRC [11, 17-24] - were among the top most differentially abundant microbes in dMMR tumor samples but were not found to be differentially abundant in pMMR tumor samples. As the GLMM had adjusted sample type, sample location (distal/proximal), and age in the model, these significant associations were less likely to be driven by these potential confounders. Indeed, we observed an enrichment of the dMMR-associated microbes regardless of sample locations (Additional file 1: Figure S3).

To validate these results, we used publicly available data from tumor and matched normal samples from 44 CRC patients [49]. Our validation analysis showed several overlapping associations of microbial genomes with respect to $\mathrm{dMMR}$ and $\mathrm{PMMR}$ in tumor and matched normal samples (Additional file 1: Tables S7, S8). dMMR tumors were found enriched for $B$. fragilis ( $p=0.02$, FDR $p=0.37)$ and Fusobacterium $(p=0.03$, FDR $p=0.37)$ while dMMR normal samples were enriched for Dorea $(p=0.03$, FDR $p=0.37)$ and an Erysipelotrichaceae bacterium ( $p=0.007$, FDR $p=0.31$ ) (Additional file 1: Figure S4). Even though these associations were not statistically significant after correcting for FDR, their trend of association overlaps with the results from the present study. Differentially abundant sOTUs between pMMR tumors versus normal included Ruminococcaceae, Faecalibacterium prausnitzii, and Bacteroides caccae, which were also differentially abundant in the present study.

\section{Proxies for hydrogen sulfide production enriched in the dMMR CRC tumors}

As sulfidogenic $F$. nucleatum and $F$. periodonticum were also significantly enriched in dMMR tumor samples, we decided to assess potential hydrogen sulfide production across groups (dMMR/pMMR, tumor/normal) by modeling hydrogen sulfide flux. We used microbial community metabolic models to predict hydrogen sulfide flux within each microbial community using MICOM. We then took the values for the hydrogen sulfide flux and calculated the average value within each group (dMMR tumor and normal, pMMR tumor and normal). The models produced a non-significant trend towards increased hydrogen sulfide flux in tumor samples (Fig. 2a). To get a more concrete measure of hydrogen sulfide production, we ran targeted metabolomics to quantify amino acid proxies (serine, homoserine, lanthionine, L-cystathionine, D-cystathionine) for hydrogen sulfide in $\mathrm{dMMR}$ and $\mathrm{PMMR}$ tumor and normal tissue samples (Fig. 2b). We observed a significant increase in lanthionine in $\mathrm{dMMR}$ tumor tissue over $\mathrm{dMMR}$ or pMMR normal tissue and PMMR tumor tissue. Homoserine and L-cystathionine were also significantly increased in both $\mathrm{dMMR}$ and pMMR tumor tissue as compared to normal-adjacent tissue. The metabolomics results suggest 


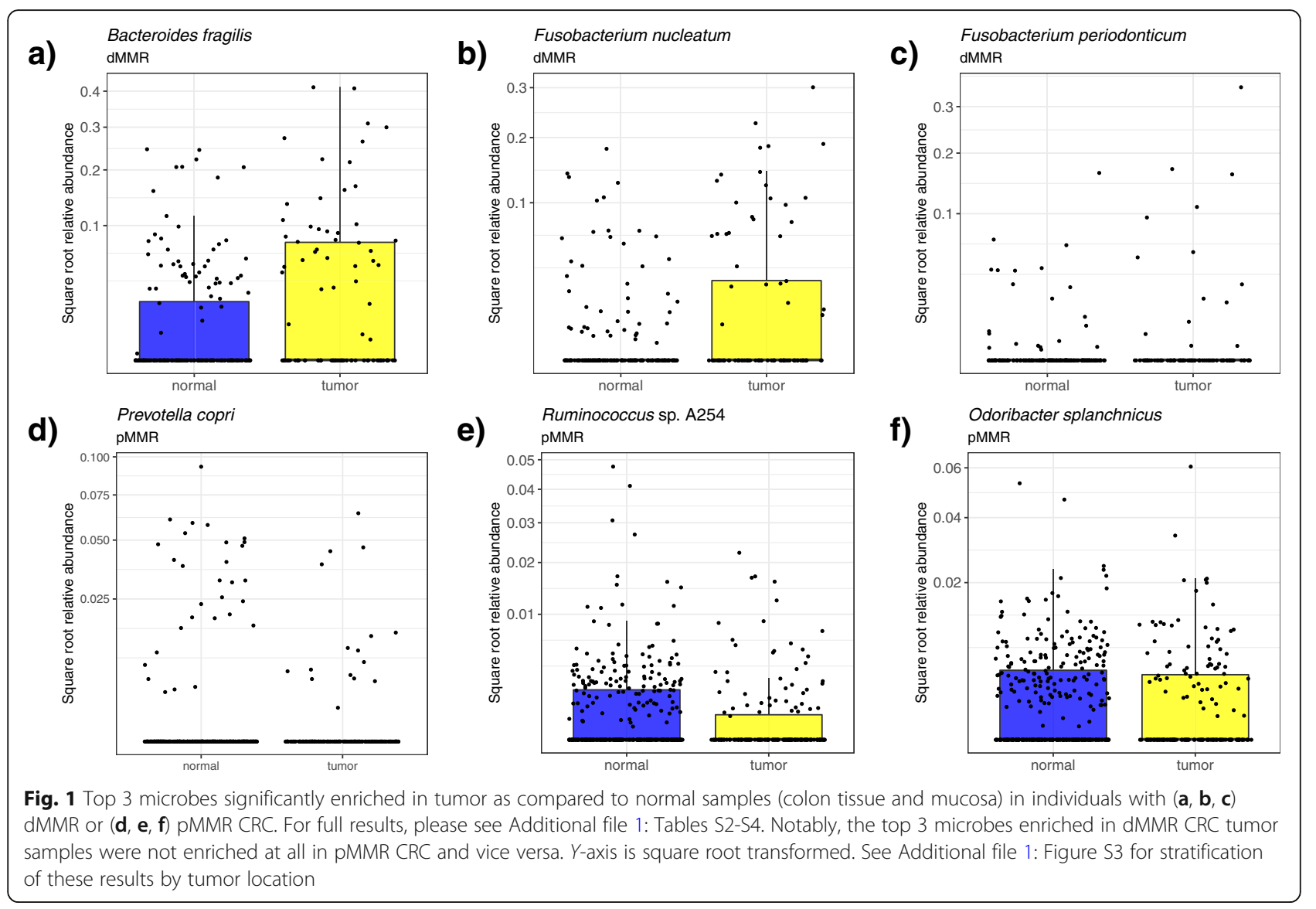

increased hydrogen sulfide production in tumor tissueparticularly in dMMR tumor tissue.

\section{dMMR and pMMR tumor and normal-adjacent microbial community predicted to be highly influenced by differing Bacteroides species}

To further assess the potential metabolic interactions between tumor and normal-adjacent microbes in relation to MMR status, we constructed two metabolic influence networks (MIN; Fig. 3) [52]. The MIN highlights each microbe's predicted influence and interactions (growth enhancing or suppressing) in relation to other microbes in the community. The major influencers (largest nodes) are generally composed of primary fermenters, such as Bacteroides or Prevotella. However, in $\mathrm{dMMR}$, the normal-adjacent community is anchored by the highly influential $B$. caccae and $B$. ovatus while in the pMMR MIN, the tumor community is anchored by D. longicatena and a Bacteroides sp. These different $\mathrm{dMMR}$ and $\mathrm{pMMR}$ communities appear to have different key species that influence the rest of the microbial community. Also of note in relation to the dMMR MIN, $F$. nucleatum and $F$. periodonticum exhibit no metabolic interactions with the other microbes in the network and therefore were not included in the network visualization.

\section{pMMR microbial community predicted to enhance suppression of Bacteroides fragilis}

The differences between the MINs for dMMR and pMMR microbial communities only relate to the tumor or normal-adjacent associated microbiota. While remarkable and noteworthy for understanding how the key species metabolically suppress or promote one another, it is nonetheless an incomplete picture of the effect of the microbial community on bacterial species growth. In order to better assess the impact of dMMR and pMMR communities as a whole on B. fragilis, we computed an interaction score and took an abundance-weighted sum of the effect of those interactions of $B$. fragilis for each microbial community. When comparing dMMR and pMMR communities, we see a statistically significant difference (Fig. 4; Wilcoxon rank sum $p<0.001$ ) in the growth suppression of $B$. fragilis, with markedly more suppression in pMMR communities where B. fragilis is not associated with CRC. This is consistent with the idea that $B$. fragilis may play a central role in dMMR but not pMMR CRC as suggested by our GLMM. 

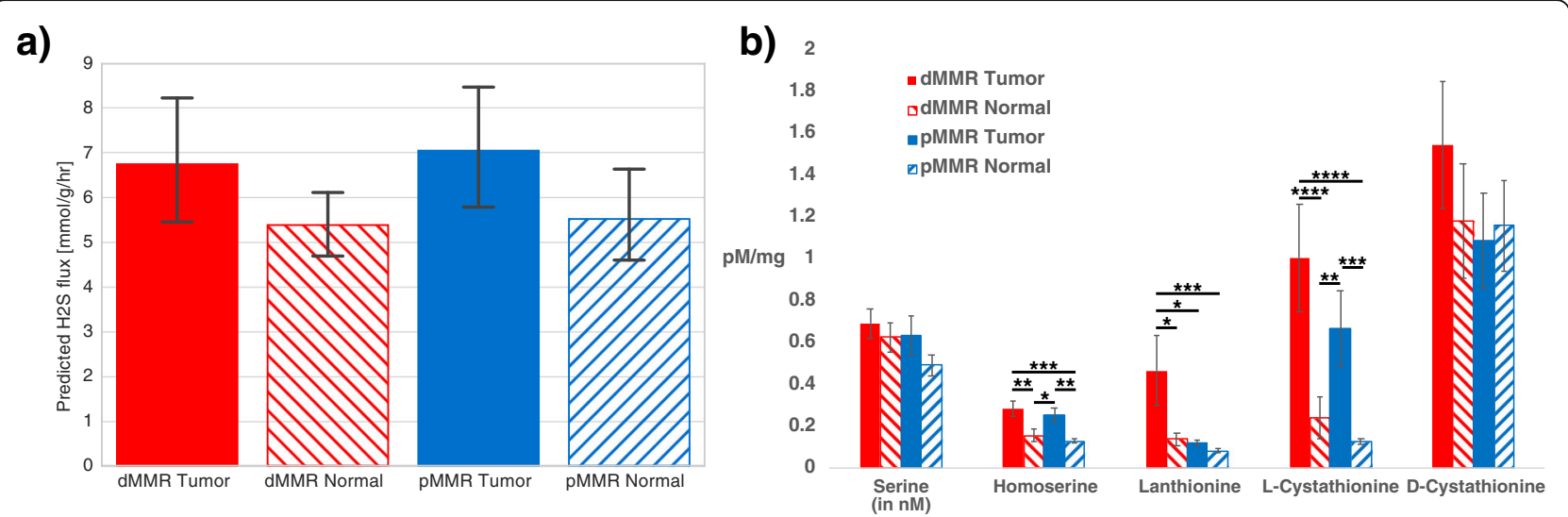

Fig. 2 a Hydrogen sulfide flux predicted based on community metabolic modeling. Flux was predicted in millimol per gram dry weight of bacteria per hour. b Amino acid proxies for hydrogen sulfide were quantified using UPLC-MS on dMMR and pMMR tumor and normal-adjacent colon tissue samples (Kruskal-Wallis followed by Dunn's Test for posthoc comparisons: ${ }^{*} p<0.05 ;{ }^{* *} p<0.0005,{ }^{* * *} p<0.0005,{ }^{* * * *} p<0.00005$ )

Given this finding and the well-established links between toxigenic B. fragilis and colorectal cancer [19, 21, 24], we next looked for the presence of the $B$. fragilis toxin (BFT) gene in $\mathrm{dMMR}$ and $\mathrm{pMMR}$ tissue and mucosa samples. Of the 22 individuals with $\mathrm{dMMR} \mathrm{CRC}$, only one was BFT positive (5\%); of 53 individuals with pMMR CRC, only five were BFT positive $(9.4 \%)$. There was no significant difference in BFT presence between individuals with $\mathrm{dMMR}$ or pMMR CRC (Chi-squared, $p=0.477$ ).

\section{Discussion}

This study integrates tumor biology and microbial ecology in a novel and powerful approach to understanding colorectal cancer. Our results indicate that MMR status is one of the strongest predictors of microbial community variance; however, few studies [32-34], to date, include MMR status in microbial community analysis of colorectal cancer. Interestingly, we also identified several differentially abundant microbes associated with dMMR but not pMMR tumor samples including $F$. nucleatum, F. periodonticum, and B. fragilis. We further validated these findings in an independent cohort [49], which underscores the importance of including MMR status in future CRC microbiome studies. We additionally characterized the predicted and actual metabolic profiles of $\mathrm{dMMR}$ and pMMR individuals in relation to hydrogen sulfide production, and we generated a network of predicted interactions within the dMMR and pMMR microbial communities.

Hydrogen sulfide has been reported to both promote and inhibit colorectal cancer [54-57]. To assess the role of hydrogen sulfide within our study, we looked for sulfidogenic bacteria, predicted hydrogen sulfide production using community metabolic models, and indirectly measured hydrogen sulfide concentrations through targeted metabolomics for amino acid proxies. We found two significantly enriched hydrogen sulfide-producing Fusobacterium species and significantly increased proxies for hydrogen sulfide in dMMR tumor samples. In the microbial influence network, both Fusobacterium species exhibited zero predicted interactions-positive or negative-with other microbes in the network. Together, this suggests that these Fusobacterium species may grow unchecked by other microbes and have the potential to produce large quantities of hydrogen sulfide.

These intriguing results lead us to speculate on the relationship between Fusobacterium species, hydrogen sulfide production, and dMMR CRC. Notably, Fusobacterium species have previously been associated with hypermethylation of MLH1, MSI, BRAF mutations, and poorly differentiated tumors $[12,22]$-all of which are characteristics of dMMR CRC [25]. Hydrogen sulfide-a cytotoxic, genotoxic gashas also been associated with CRC $[54,55]$, although there have been conflicting reports on its role $[56,57]$. A recent report indicates that colon cancer cells may respond to hydrogen sulfide in a bell-shaped dose-dependent manner: at high concentrations, hydrogen sulfide inhibits the proliferation of cancer cells, while at lower concentrations, hydrogen sulfide can stimulate the proliferation of cancer cells $[57,58]$. In dMMR, if high levels of hydrogen sulfide (and hydrogen sulfide producers) inhibit cancer proliferation, then we would expect individuals with dMMR to present with earlier-stage cancer-which is indeed the case in our cohort and other reported cohorts [25].

Epidemiologically, it is worth noting that dMMR CRC has also been associated with lower recurrence rates and a better prognosis [25]. In seeming opposition to these findings are studies showing that F. nucleatum can potentiate tumorigenesis and that $F$. nucleatum-associated CRCs have a worse prognosis [11, 12]. However, these findings are not contradictory with our data. A more 


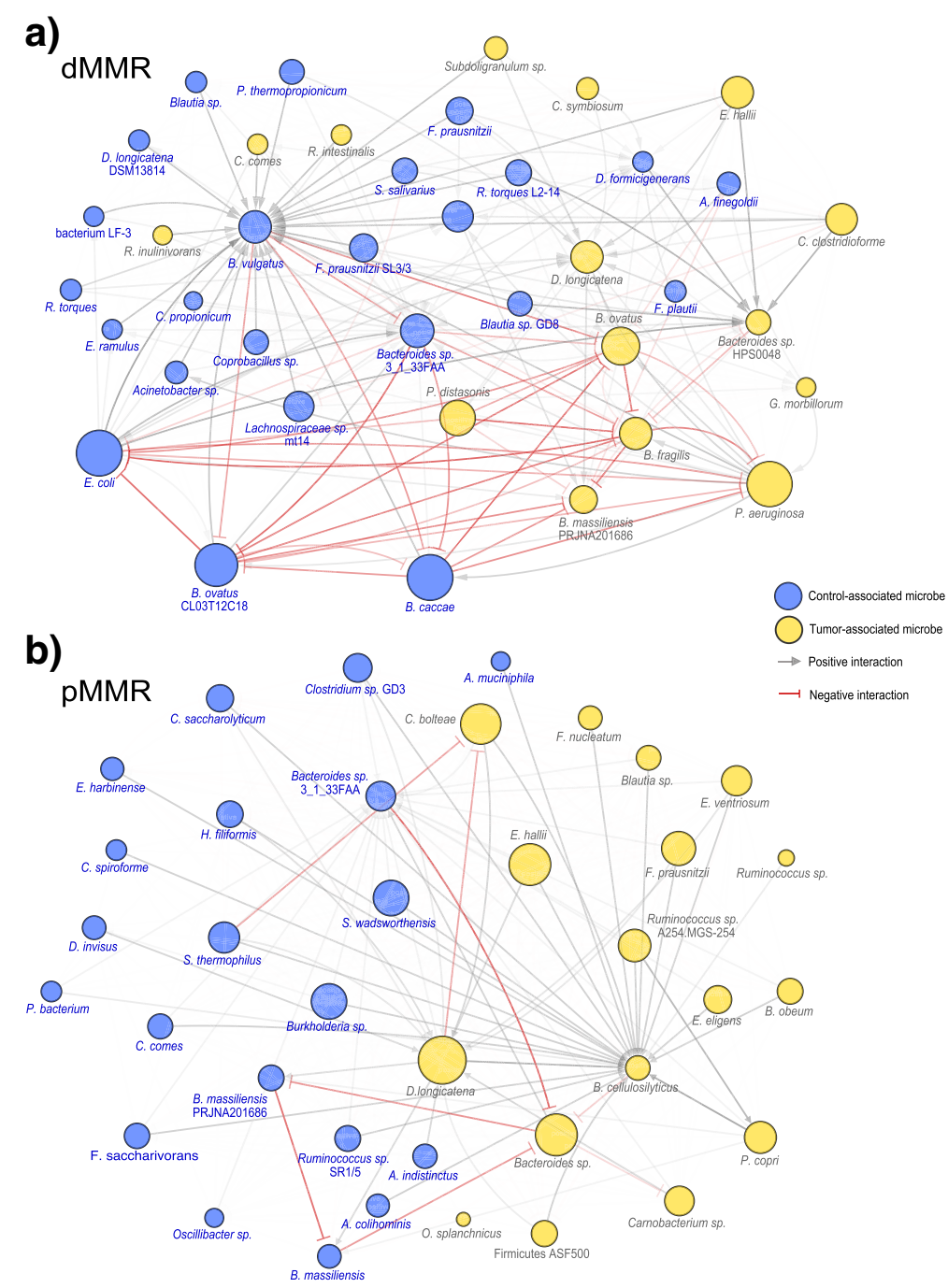

Fig. 3 Microbial influence networks for $\mathbf{a}$ dMMR and $\mathbf{b}$ pMMR microbial communities. Node size indicates a microbe's metabolic influence over other microbes. Edges, which are directional and weighted according to the magnitude of their influence, indicate how one microbe affects the growth rate of another. Grey edges indicate a positive interaction, i.e., predicted increase in growth when paired, while red edges indicate a negative interaction, i.e., predicted suppression in growth when paired

detailed examination of the effects of location (Additional file 1, Tables S5 and S6) shows that Fusobacterium is associated with the proximal colon in both dMMR and pMMR patients. This raises a subtle, but important, point. Fusobacterium-associated pMMR tumors are very likely to be found in the proximal colon alongside normal-adjacent tissue that is also enriched for Fusobacterium. Stated another way, while pMMR tumors are not especially associated with Fusobacterium, the proximal colon is. (In contrast, dMMR tumors show enrichment for Fusobacterium that goes beyond the effect of location in the colon.) When put into context with other epidemiological findings that identify right-sided (proximal) colon cancer to have lower overall survival [59], certain inferences come to light. Where right-sided AMMR CRCs have a relatively better prognosis, right-sided pMMR CRCs have a worse one. This would then allow us to make sense of both the overall lower survival in right-sided CRC [59] and the results indicating $F$. nucleatum-associated CRCs have a worse prognosis $[11,12]$. In sum, the prognosis of $F$. nucleatu$m$-associated CRCs is likely be dependent upon both location and tumor MMR status, and our study highlights the importance of evaluating these covariates simultaneously when determining tumor prognosis.

Besides Fusobacterium, B. fragilis was also found to be significantly enriched in dMMR tumor samples. Toxigenic B. fragilis has well-established and causative links to inflammation and CRC [19, 21, 24], and inflammation has been linked to hypermethylation [60]. Our own metabolic 


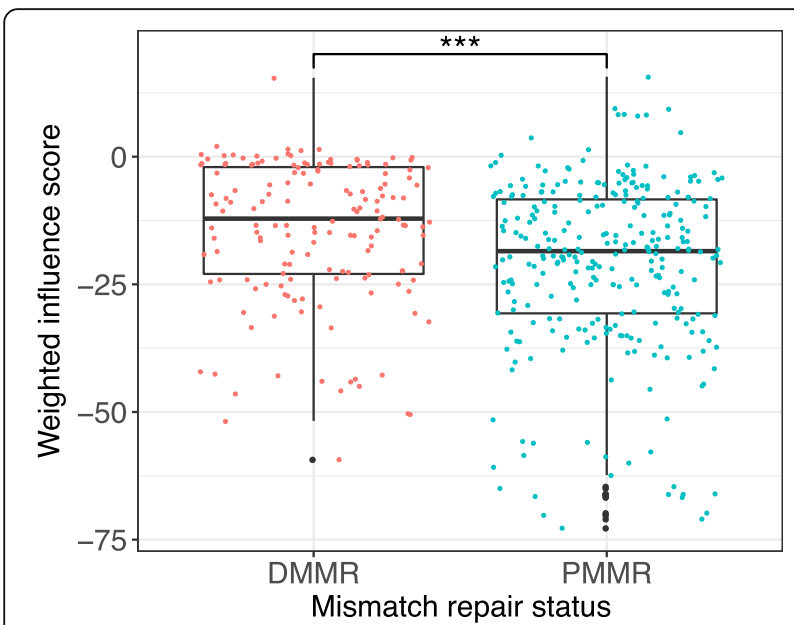

Fig. 4 Predicted influence of other microbes (weighted influence score) on $B$. fragilis growth, stratified by MMR status. A negative influence score indicates microbial community suppression of $B$. fragilis growth. $B$. fragilis is significantly more suppressed in $\mathrm{pMMR}$ microbial communities (tumor and normal) as compared to dMMR microbial communities (Wilcoxon rank sum test $p<0.001$ )

modeling reflects a metabolic basis for higher ratios of $B$. fragilis in dMMR communities, and greater metabolic suppression in pMMR. We tested dMMR and pMMR tissue and mucosa samples for the presence of the $B$. fragilis toxin (BFT) gene but did not find a significant difference in the presence of the BFT gene between dMMR and pMMR individuals. Given these results, it is unclear what the significance of toxigenic $B$. fragilis is in the dMMR tumor samples.

Overall, our study demonstrates the importance and value in considering tumor biology (MMR status) and ecological interactions when evaluating microbial community data. Our work is primarily descriptive and incorporates host clinical features, microbiome, metabolome, and modeling data. While we make speculations based on these data, future prospective and mechanistic studies are needed to test these ideas. We also recognize that selecting sequenced genomes available in the database to represent $16 \mathrm{~S}$ rRNA sOTUs cannot fully replace metagenomic sequencing given well-known strain-to-strain variation in gene content. However, these variations between strains are often largely in secondary metabolite pathways, rather than core metabolic function, which is the main target of our modeling analysis. Differences in secondary metabolite pathways (i.e., non-core genome within a species) are commonly associated with functional adaptations to various environmental niches [61].

Another limitation of this study is our inability to attribute a source to metabolomic data. Hydrogen sulfide and its amino acid proxies can be produced by both humans and bacteria. Thus, the enriched hydrogen sulfide we detect in dMMR tumor samples could potentially be attributed to increased hydrogen sulfide production within tumor tissue, and indeed, this has been reported [57]. If this was the solely case here however, we might expect to see similar increases in hydrogen sulfide in pMMR tumors-most of which are later in stage than dMMR tumors. We did not see this, suggesting that it is feasible that the increased hydrogen sulfide production in dMMR tumors is coming from an exogenous (microbial) source. Notably, microbially produced hydrogen sulfide can be generated from multiple pathways including the respiration of dietary taurine and sulfate as well as the degradation of sulfomucins. The amino acid proxies we use to assess hydrogen sulfide production only capture some, but not all of these potential pathways, so we may have underestimated hydrogen sulfide production.

Finally, the field of genome-scale metabolic modeling has only recently encompassed tools for community metabolic analyses [62], and many of the tools [51, 52, 63] are sensitive to the underlying quality of the metabolic models [64, 65]. Models vary greatly depending on the presence and accuracy of genome annotations which will generally improve over time. Future work aimed at understanding and verifying microbial dynamics in relation to MMR status or other CRC subtypes could dramatically improve our ability to define, predict, prevent, and treat colorectal cancers.

\section{Conclusions}

This study provides a novel framework in which to examine colorectal cancer:

1. Host-microbe interactions: Tumor MMR status strongly predicted microbial community variance and was associated with distinct microbial, metabolic, and interaction profiles. Our approach incorporating tumor MMR status, microbiome, metabolome, and modeling data allowed us unique insights into the role of hydrogen sulfide and hydrogen sulfide producers within the dMMR microbial community. Tumor biology (e.g., MMR status) and microbial ecology are inextricably linked, and it is critical that future studies account for both in order to understand and more precisely classify the many pathways to CRC.

2. Microbe-microbe interactions: Microbial influence networks provided in silico predictions of microbial interactions that aligned with in vivo metabolomics data: Enrichment of sulfidogenic F. nucleatum and significantly higher hydrogen sulfide production in dMMR CRC, and depletion of $B$. fragilis and significantly higher suppression in pMMR CRC. The validation of in vivo findings and in silico modeling provides support for a future of precision medicine tools that can accurately predict disease and the potential effects of prophylactic or 
therapeutic interventions on the microbiome. Microbes act within communities, and understanding and predicting these interactions will be key to developing targeted mechanisms to help prevent or treat colorectal cancer.

\section{Additional files}

Additional file 1: Figure S1. Unweighted Unifrac distances between tumor and normal-associated microbiota in $\mathrm{dMMR}$ and pMMR. Figure S2. Venn diagram highlighting number of microbes that overlap between tumor and normal samples in relation to MMR status ( $\mathrm{dMMR}=$ red font, $\mathrm{pMMR}=$ green font, red circles = tumor samples, blue circles =normal samples). Figure S3. Microbes significantly enriched in tumor as compared to normal samples (colon tissue and mucosa) in individuals with dMMR CRC: a) Bacteroides fragilis and b) Fusobacterium nucleatum. Figure S4. Differentially abundant OTUs between patient-matched tumor and normal samples in individuals with $\mathrm{AMMR} C R C$ from an independent cohort. Table S1. Table S1: List of PATRIC model IDs and associated sOTUs. Table S2. Microbes identified as differentially abundant in tumor as compared to normal samples (tissue and mucosa) from individuals with dMMR or PMMR CRC. Table S3. SOTUs enriched in tumor samples (colon tissue and mucosa) as compared to normal adjacent samples in individuals with AMMR CRC. Table S4. sOTUs enriched in tumor samples (colon tissue and mucosa) as compared to normal adjacent samples in individuals with pMMR CRC. Table S5. sOTUs enriched in the proximal or distal colon (colon tissue and mucosa) of individuals with dMMR CRC. Table S6. sOTUs enriched in the proximal or distal colon (colon tissue and mucosa) of individuals with pMMR CRC. Table S7. Differentially abundant microbes in individuals with $\mathrm{AMMR} C R C$ from an independent cohort. Table S8. Differentially abundant microbes in individuals with pMMR CRC from an independent cohort. (PDF $2790 \mathrm{~kb}$ )

Additional file 2: SOTU read counts by sample. (TSV $5546 \mathrm{~kb}$ )

Additional file 3: sOTU ID and taxonomy. (TXT $654 \mathrm{~kb}$ )

Additional file 4: SOTU ID and sequences. (FASTA $2042 \mathrm{~kb}$ )

\section{Acknowledgements}

We would first like to thank the patients who volunteered for this study. We also thank the many other individuals who made this work possible including members of the Mayo Clinic Microbiome Laboratory, study coordinators, students, colorectal surgeons, program directors, and pathology assistants. We also specially acknowledge Donna Felmlee Devine and Caitlin Foss-Baumgard for their assistance with patient records in relation to this study.

\section{Funding}

We gratefully acknowledge the following funding sources: $\mathrm{NIH}$ (R01CA179243; N.C. and V.L.H. and R01CA170357; L.B.), the Mayo Clinic Center for Cell Signaling in Gastroenterology (NIDDK P30DK084567), the Mayo Clinic Metabolomics Resource Core Pilot and Feasibility Award (U24DK100469), the Fred C. Andersen Foundation (H.N. and N.C.), the Mayo Clinic Center for Individualized Medicine, The Randy Shaver Cancer Research and Community Fund (R.B.), the Minnesota Partnership for Biotechnology and Medical Genomics (R.B.), and the Alfred P. Sloan Foundation (R.B.). O.R.A thanks the financial support coming from the National Institute of Genomic Medicine (INMEGEN) to develop the computational tool used for the microbiome analysis (MICOM).

\section{Availability of data and materials}

The 165 datasets generated and/or analyzed during the current study are available in the NCBI Short Read Archive, under BioProject PRJNA445346: https://www.ncbi.nlm.nih.gov/bioproject/445346, PRJNA284355: https:// www.ncbi.nlm.nih.gov/sra/PRJNA284355, and Additional files 2, 3, and 4. Metabolomics data is available here: https://figshare.com/articles/ AminoAcid_Metabolomics_ForPublicSharing_xlsx/7065053

\section{Authors' contributions}

$N C, H N, B A W, V L H$, and PJ conceived of various aspects of this project. VLH, $J Y, G K, K L, L A B, D L$, and $H N$ were involved in the sample collection and processing for microbial sequencing. PJ, VLH, JC, MM, JS, CD, and OR-A were involved in the analysis of 165 and community metabolic modeling data and figure generation. X-MP, TD, JG, and VLH were involved in metabolomic sample prep and analysis. JR provided critical insights on microbial metabolite production pathways. AJF and SNT were involved in MMR status identification via immunohistochemistry. SP and RB provided analysis of the independent cohort. All authors read and approved the final manuscript.

\section{Ethics approval and consent to participate}

This study was performed with the approval of the Mayo Clinic Institutional Review Board (IRB\# 14-007237 and IRB\# 622-00) in accordance with the principles of the Declaration of Helsinki. Written informed consent was obtained from all individuals in the study.

\section{Consent for publication}

Not applicable

\section{Competing interests}

The authors declare that they have no competing interests.

\section{Publisher's Note}

Springer Nature remains neutral with regard to jurisdictional claims in published maps and institutional affiliations.

\section{Author details}

${ }^{1}$ Department of Veterinary Preventive Medicine, The Ohio State University College of Veterinary Medicine, Columbus, OH, USA. ${ }^{2}$ Microbiome Program, Center for Individualized Medicine, Mayo Clinic, Rochester, MN, USA. ${ }^{3}$ Division of Surgical Research, Department of Surgery, Mayo Clinic, Rochester, MN, USA. ${ }^{4}$ Department of Health Sciences Research, Mayo Clinic, Rochester, MN, USA. ${ }^{5}$ Center for Individualized Medicine, Mayo Clinic, Rochester, MN, USA. ${ }^{6}$ Department of Genetics, Cell Biology, and Development, University of Minnesota, Minneapolis, MN, USA. 'Division of Laboratory Medicine and Pathology, Mayo Clinic, Rochester, MN, USA. ${ }^{8}$ Carl R. Woese Institute for Genomic Biology, Department of Animal Sciences, Division of Nutritional Sciences, University of Illinois, Urbana-Champaign, IL, USA. ${ }^{9}$ Human Systems Biology Laboratory, National Institute of Genomic Medicine, Mexico City, Mexico. ${ }^{10}$ Coordinación de la Investigación Científica, Red de Apoyo a la Investigación, UNAM, Mexico City, Mexico. ${ }^{11}$ Mayo Clinic Metabolomics Core Laboratory, Mayo Clinic, Rochester, MN, USA. ${ }^{12}$ Division of Gastroenterology and Hepatology, Mayo Clinic, Rochester, MN, USA.

${ }^{13}$ Division of Colon and Rectal Surgery, Department of Surgery, Mayo Clinic, Rochester, MN, USA

Received: 13 June 2018 Accepted: 8 October 2018

Published online: 31 October 2018

\section{References}

1. Flemer B, Lynch DB, Brown JMR, Jeffery IB, Ryan FJ, Claesson MJ, et al. Tumour-associated and non-tumour-associated microbiota in colorectal cancer. Gut. 2017;66:633-43. https://doi.org/10.1136/gutjnl-2015-309595.

2. Chen W, Liu F, Ling Z, Tong X, Xiang C. Human intestinal lumen and mucosa-associated microbiota in patients with colorectal cancer. PLoS One. 2012:7:e39743.

3. Zackular JP, Baxter NT, Chen GY, Schloss PD. Manipulation of the Gut Microbiota Reveals Role in Colon Tumorigenesis mSphere 2016;1:e00001e00015. doi:https://doi.org/10.1128/mSphere.00001-15.

4. Ahn J, Sinha R, Pei Z, Dominianni C, Wu J, Shi J, et al. Human gut microbiome and risk for colorectal cancer. J Natl Cancer Inst. 2013;105:1907-11.

5. Arthur JC, Gharaibeh RZ, Muhlbauer M, Perez-Chanona E, Uronis JM, McCafferty J, et al. Microbial genomic analysis reveals the essential role of inflammation in bacteria-induced colorectal cancer. Nat Commun. 2014;5: 4724. https://doi.org/10.1038/ncomms5724.

6. Brennan CA, Garrett WS. Gut microbiota, inflammation, and colorectal cancer. Annu Rev Microbiol. 2016;70:395-411. https://doi.org/10.1146/ annurev-micro-102215-095513. 
7. Marchesi JR, Dutilh BE, Hall N, Peters WH, Roelofs R, Boleij A, et al. Towards the human colorectal cancer microbiome. PLoS One. 2011;6:e20447. https:// doi.org/10.1371/journal.pone.0020447.

8. Sobhani I, Tap J, Roudot-Thoraval F, Roperch JP, Letulle S, Langella P, et al. Microbial dysbiosis in colorectal cancer (CRC) patients. PLoS One. 2011;6: e16393.

9. Louis P, Hold GL, Flint HJ. The gut microbiota, bacterial metabolites and colorectal cancer. Nat Rev Microbiol. 2014;12:661-72.

10. Castellarin M, Warren RL, Freeman JD, Dreolini L, Krzywinski M, Strauss J, et al. Fusobacterium nucleatum infection is prevalent in human colorectal carcinoma. Genome Res. 2012;22:299-306.

11. Kostic AD, Chun E, Robertson L, Glickman JN, Gallini CA, Michaud M, et al. Fusobacterium nucleatum potentiates intestinal tumorigenesis and modulates the tumor-immune microenvironment. Cell Host Microbe. 2013;14:207-15.

12. Mima K, Nishihara R, Qian ZR, Cao Y, Sukawa Y, Nowak JA, et al. Fusobacterium nucleatum in colorectal carcinoma tissue and patient prognosis. Gut. 2016:65:1973-80.

13. Flanagan L, Schmid J, Ebert M, Soucek P, Kunicka T, Liska V, et al. Fusobacterium nucleatum associates with stages of colorectal neoplasia development, colorectal cancer and disease outcome. Eur J Clin Microbiol Infect Dis. 2014;33:1381-90.

14. Kostic AD, Gevers D, Pedamallu CS, Michaud M, Duke F, Earl AM, et al. Genomic analysis identifies association of Fusobacterium with colorectal carcinoma. Genome Res. 2012;22:292-8.

15. Drewes JL, White JR, Dejea CM, Fathi P, lyadorai T, Vadivelu J, et al. Highresolution bacterial 16S rRNA gene profile meta-analysis and biofilm status reveal common colorectal cancer consortia. NPJ Biofilms Microbiomes. 2017;3:34.

16. Sinha R, Abu-Ali G, Vogtmann E, Fodor AA, Ren B, Amir A, et al. Assessment of variation in microbial community amplicon sequencing by the Microbiome Quality Control (MBQC) project consortium. Nat Biotechnol. 2017;35:1077-86. https://doi.org/10.1038/nbt.3981.

17. Rubinstein MR, Wang X, Liu W, Hao Y, Cai G, Han YW. Fusobacterium nucleatum promotes colorectal carcinogenesis by modulating E-cadherin/ $\beta$ catenin signaling via its FadA adhesin. Cell Host Microbe. 2013;14:195-206.

18. Yang $Y$, Weng $W$, Peng J, Hong L, Yang L, Toiyama $Y$, et al. Fusobacterium nucleatum increases proliferation of colorectal cancer cells and tumor development in mice by activating TLR4 signaling to NFKB, upregulating expression of microRNA-21. Gastroenterology. 2016;152:851-866.e24. https:// doi.org/10.1053/j.gastro.2016.11.018.

19. Housseau F, Sears CL. Enterotoxigenic Bacteroides fragilis (ETBF)-mediated colitis in min (Apc+/-) mice: a human commensal-based murine model of colon carcinogenesis. Cell Cycle. 2010;9:3-5.

20. Purcell RV, Pearson J, Aitchison A, Dixon L, Frizelle FA, Keenan J. Colonization with enterotoxigenic Bacteroides fragilis is associated with early-stage colorectal neoplasia. PLoS One. 2017;12:e0171602.

21. Chung L, Thiele Orberg E, Geis AL, Chan JL, Fu K, DeStefano Shields CE, et al. Bacteroides fragilis toxin coordinates a pro-carcinogenic inflammatory cascade via targeting of colonic epithelial cells. Cell Host Microbe. 2018;23: 203-214.e5. https://doi.org/10.1016/j.chom.2018.01.007.

22. Koi M, Okita Y, Carethers JM. Fusobacterium nucleatum infection in colorectal cancer: linking inflammation, DNA mismatch repair and genetic and epigenetic alterations. J Anus, Rectum Colon. 2018;2:37-46. https://doi. org/10.23922/jarc.2017-055

23. Abed J, Emgård JEM, Zamir G, Faroja M, Almogy G, Grenov A, et al. Fap2 mediates fusobacterium nucleatum colorectal adenocarcinoma enrichment by binding to tumor-expressed Gal-GalNAc. Cell Host Microbe. 2016;20:215-25.

24. Dejea CM, Fathi P, Craig JM, Boleij A, Taddese R, Geis AL, et al. Patients with familial adenomatous polyposis harbor colonic biofilms containing tumorigenic bacteria. Science (80- ). 2018;359:592-7.

25. Richman S. Deficient mismatch repair: read all about it (review). Int J Oncol. 2015:47:1189-202.

26. French AJ, Sargent DJ, Burgart LJ, Foster NR, Kabat BF, Goldberg R, et al. Prognostic significance of defective mismatch repair and BRAF V600E in patients with colon cancer. Clin Cancer Res. 2008;14:3408-15. https://doi. org/10.1158/1078-0432.CCR-07-1489.

27. Guinney J, Dienstmann R, Wang X, De Reyniès A, Schlicker A, Soneson C, et al. The consensus molecular subtypes of colorectal cancer. Nat Med. 2015; 21:1350.

28. Mårtensson A, Oberg A, Jung A, Cederquist K, Stenling R, Palmqvist R. Betacatenin expression in relation to genetic instability and prognosis in colorectal cancer. Oncol Rep. 2007;17:447-52.
29. Morkel M, Riemer P, Bläker H, Sers C. Similar but different: distinct roles for KRAS and BRAF oncogenes in colorectal cancer development and therapy resistance. Oncotarget. 2015;6:20785-800. https://doi.org/10.18632/ oncotarget.4750.

30. Sweetser S, Jones A, Smyrk TC, Sinicrope FA. Sessile serrated polyps are precursors of colon carcinomas with deficient DNA mismatch repair. Clin Gastroenterol Hepatol. 2016;14:1056-9.

31. Dejea CM, Wick EC, Hechenbleikner EM, White JR, Mark Welch JL, Rossetti $B J$, et al. Microbiota organization is a distinct feature of proximal colorectal cancers. Proc Natl Acad Sci. 2014;111:18321-6. https://doi.org/10.1073/pnas. 1406199111.

32. Purcell RV, Visnovska M, Biggs PJ, Schmeier S, Frizelle FA. Distinct gut microbiome patterns associate with consensus molecular subtypes of colorectal cancer. Sci Rep. 2017;7:11590

33. Belcheva A, Irrazabal T, Robertson SJ, Streutker C, Maughan H, Rubino S, et al. Gut microbial metabolism drives transformation of msh2-deficient colon epithelial cells. Cell. 2014:158:288-99.

34. Lennard KS, Goosen RW, Blackburn JM. Bacterially-associated transcriptional remodelling in a distinct genomic subtype of colorectal cancer provides a plausible molecular basis for disease development. PLoS One. 2016;11: e0166282. https://doi.org/10.1371/journal.pone.0166282.

35. Hale VL, Jeraldo P, Mundy M, Yao J, Keeney G, Scott N, et al. Synthesis of multi-omic data and community metabolic models reveals insights into the role of hydrogen sulfide in colon cancer. Methods. 2018. https://doi.org/10. 1016/j.ymeth.2018.04.024.

36. Chen J, Ryu E, Hathcock M, Ballman K, Chia N, Olson JE, et al. Impact of demographics on human gut microbial diversity in a US Midwest population. PeerJ. 2016;4:e1514. https://doi.org/10.7717/peerj.1514.

37. Hale VL, Chen J, Johnson S, Harrington SC, Yab TC, Smyrk TC, et al. Shifts in the fecal microbiota associated with adenomatous polyps. Cancer Epidemiol Prev Biomarkers. 2017;26:85-94

38. Callahan BJ, McMurdie PJ, Rosen MJ, Han AW, Johnson AJA, Holmes SP. DADA2: high-resolution sample inference from Illumina amplicon data. Nat Methods. 2016:13:581-3.

39. Wang Q, Garrity GM, Tiedje JM, Cole JR. Naïve Bayesian classifier for rapid assignment of rRNA sequences into the new bacterial taxonomy. Appl Environ Microbiol. 2007;73:5261-7.

40. Quast C, Pruesse E, Yilmaz P, Gerken J, Schweer T, Yarza P, et al. The SILVA ribosomal RNA gene database project: improved data processing and webbased tools. Nucleic Acids Res. 2013;41:D590-6.

41. Kopylova E, Noé L, Touzet H. SortMeRNA: fast and accurate filtering of ribosomal RNAs in metatranscriptomic data. Bioinformatics. 2012;28:3211-7.

42. Nawrocki EP, Eddy SR. Infernal 1.1: 100-fold faster RNA homology searches. Bioinformatics. 2013;29:2933-5.

43. Price MN, Dehal PS, Arkin AP. FastTree 2 - approximately maximumlikelihood trees for large alignments. PLoS One. 2010;5:e9490.

44. Lozupone C, Knight R. UniFrac: a new phylogenetic method for comparing microbial communities. Appl Environ Microbiol. 2005;71:8228-35. https://doi. org/10.1128/AEM.71.12.8228-8235.2005.

45. McMurdie PJ, Holmes S. Phyloseq: an R package for reproducible interactive analysis and graphics of microbiome census data. PLoS One. 2013:8:e61217. https://doi.org/10.1371/journal.pone.0061217.

46. Oksanen J, Kindt R, Legendre P, O'Hara B, Simpson GL, Solymos PM, et al. The vegan package. Community Ecol Packag. 2008:190. https://doi.org/10. 4135/9781412971874.n145.

47. Bolker BM, Brooks ME, Clark CJ, Geange SW, Poulsen JR, Stevens MHH, et al. Generalized linear mixed models: a practical guide for ecology and evolution. Trends Ecol Evol. 2009;24:127-35. https://doi.org/10.1016/j.tree.2008.10.008.

48. Brooks ME, Kristensen K, van Benthem KJ, Magnusson A, Berg CW, Nielsen A, et al. glmmTMB balances speed and flexibility among packages for zeroinflated generalized linear mixed modeling. R J 2017:9:378-400.

49. Burns MB, Montassier E, Abrahante J, Priya S, Niccum DE, Khoruts A, et al. Colorectal cancer mutational profiles correlate with defined microbial communities in the tumor microenvironment. PLoS Genet. 2018;14:090795. https://doi.org/10.1371/journal.pgen.1007376.

50. Orth JD, Thiele I, Palsson B $\varnothing$. What is flux balance analysis? Nat Biotechnol. 2010;28:245-8. https://doi.org/10.1038/nbt.1614.

51. Mendes-Soares H, Mundy M, Soares LM, Chia N. MMinte: an application for predicting metabolic interactions among the microbial species in a community. BMC Bioinformatics. 2016:17:343. https://doi.org/10.1186/ s12859-016-1230-3. 
52. Sung J, Kim S, Cabatbat JJT, Jang S, Jin Y-S, Jung GY, et al. Global metabolic interaction network of the human gut microbiota for context-specific community-scale analysis. Nat Commun. 2017;8:15393. https://doi.org/10. 1038/ncomms15393.

53. Shannon P, Markiel A, Ozier O, Baliga NS, Wang JT, Ramage D, et al. Cytoscape: a software environment for integrated models of biomolecular interaction networks. Genome Res. 2003;13:2498-504.

54. Attene-Ramos MS, Nava GM, Muellner MG, Wagner ED, Plewa MJ, Gaskins HR. DNA damage and toxicogenomic analyses of hydrogen sulfide in human intestinal epithelial FHs 74 int cells. Environ Mol Mutagen. 2010;51:304-14.

55. Wolf PG, Parthasarathy G, Chen J, O'Connor HM, Chia N, Bharucha AE, et al. Assessing the colonic microbiome, hydrogenogenic and hydrogenotrophic genes, transit and breath methane in constipation. Neurogastroenterol Motil. 2017;29:1-9.

56. Lee ZW, Zhou J, Chen CS, Zhao Y, Tan CH, Li L, et al. The slow-releasing hydrogen sulfide donor, GYY4137, exhibits novel anti-cancer effects in vitro and in vivo. PLoS One. 2011;6:e21077.

57. Hellmich MR, Coletta C, Chao C, Szabo C. The therapeutic potential of cystathionine $\beta$-synthetase/hydrogen sulfide inhibition in cancer. Antioxid Redox Signal. 2015;22:424-48. https://doi.org/10.1089/ars.2014.5933.

58. Cai W, Wang M, Ju L, Wang C, Zhu Y. Hydrogen sulfide induces human colon cancer cell proliferation: role of Akt, ERK and p21. Cell Biol Int. 2010; 34:565-72. https://doi.org/10.1042/CB120090368.

59. Lim DR, Kuk JK, Kim T, Shin EJ. Comparison of oncological outcomes of right-sided colon cancer versus left-sided colon cancer after curative resection. Med (United States). 2017;96:e8241.

60. Maiuri AR, Peng M, Sriramkumar S, Kamplain CM, DeStefano Shields CE, Sears $C L$, et al. Mismatch repair proteins initiate epigenetic alterations during inflammation-driven tumorigenesis. Cancer Res. 2017;77:3467-78.

61. Lind AL, Wisecaver JH, Lameiras C, Wiemann P, Palmer JM, Keller NP, et al. Drivers of genetic diversity in secondary metabolic gene clusters within a fungal species. PLoS Biol. 2017;15:e2003583.

62. Magnúsdóttir $\mathrm{S}$, Thiele I. Modeling metabolism of the human gut microbiome. Curr Opin Biotechnol. 2018;51:90-6

63. Benedict MN, Mundy MB, Henry CS, Chia N, Price ND. Likelihood-based gene annotations for gap filling and quality assessment in genome-scale metabolic models. PLoS Comput Biol. 2014:10:e1003882.

64. Magnúsdóttir S, Heinken A, Kutt L, Ravcheev DA, Bauer E, Noronha A, et al. Generation of genome-scale metabolic reconstructions for 773 members of the human gut microbiota. Nat Biotechnol. 2017;35:81-9.

65. Wattam AR, Abraham D, Dalay O, Disz TL, Driscoll T, Gabbard JL, et al. PATRIC, the bacterial bioinformatics database and analysis resource. Nucleic Acids Res. 2014;42:D581-91.

Ready to submit your research? Choose BMC and benefit from:

- fast, convenient online submission

- thorough peer review by experienced researchers in your field

- rapid publication on acceptance

- support for research data, including large and complex data types

- gold Open Access which fosters wider collaboration and increased citations

- maximum visibility for your research: over $100 \mathrm{M}$ website views per year

At $\mathrm{BMC}$, research is always in progress.

Learn more biomedcentral.com/submissions 